\title{
Proposition d'un protocole de prélèvement pour les spermocultures
}

\author{
M.C. PINATEL*, P. BOUCHER**, Y. GILLE**, F. PINATEL*** \\ *Laboratoire de Biologie de la Reproduction. Hôpital E.Herriot Place d'Arsonval 69003 \\ Lyon ; **Laboratoire de Microbiologie de l'Hôpital de l'Antiquaille Lyon ; ***Faculté des \\ Arts Plastiques de Saint-Etienne
}

\section{RESUME}

Nous proposons un protocole de recueil du sperme pour examen bactériologique visant à éclairer et responsabiliser le patient quant à la nécessité d'une bonne asepsie. Ce protocole réduit notablement le nombre des prélèvements contaminés par des germes de voisinage et, par conséquent, le nombre d'examens ininterprétables qui doivent être renouvelés. Le mode de recueil dans de bonnes conditions est également recommandé pour les prélèvements avant PMA.

Mots-clés : sperme, spermoculture, recueil du sperme.

\section{INTRODUCTION}

La recherche d'une cause à l'hypofertilité masculine aboutit souvent à la mise en évidence d'un processus infectieux, ou de ses sequelles, au niveau des voies spermatiques ou des glandes annexes. Cette infection peut être reflétée par la présence de germes dans le sperme.

La pratique des procréations médicalement assistées (PMA) impose la vérification de l'innocuité bactériologique du sperme, pour la patiente si le sperme est déposé dans la cavité utérine (insémination artificielle), pour les cultures embyronnaires s'il s'agit d'une fécondation in vitro.

La spermoculture qui permet la recherche de germes est d'interprétation souvent dis- cutable dans la mesure où l'éjaculat peut être contaminé lors du prélèvement par masturbation. Les germes mis en évidence dans l'échantillon de sperme peuvent en effet provenir :

- soit du liquide séminal, révélant une infection latente ou patente de l'appareil génital ; ce sont ces germes qui nous intéressent et qui justifient la mise en œuvre d'un traitement ;

- soit d'une contamination par la flore uréthrale ;

- soit d'une souillure par la flore cutanéomuqueuse du gland, de la zone périgénitale ou des mains.

Nous avons essayé d'évaluer la part de ces deux dernières sources grâce à deux protocoles distincts [1] : examen bactériologique de la flore uréthrale physiologique ; pratique d'une asepsie aussi rigoureuse que possible au moment du recueil du sperme.

\section{EXAMEN BACTERIOLOGIQUE DE LA FLORE URETHRALE}

\section{Matériel et Méthodes}

Les 10 premiers millilitres (environ) de la première miction matinale ont été recueillis chez 31 hommes âgés de 15 à 75 ans, en bonne santé, appartenant au personnel du laboratoire de microbiologie ou à leur famille. Onze individus ayant effectué 2 ou 3 prélèvements, nous avons disposé au total de 46 échantillons. Aucun individu n'avait subi 
de traitement par les antibiotiques dans le mois qui a précédé le prélèvement urinaire.

Le compte de germes a été effectué par la technique à l"URICULT" (Orion Diagnostica). Les prélèvements ont été ensemencés sur des milieux de cultures appropriés et les germes ont été identifiés par les méthodes de routine du laboratoire de microbiologie.

\section{Résultats}

Le compte de germes a été inférieur à $10^{3} / \mathrm{ml}$ dans 41 échantillons, de $10^{3} / \mathrm{ml}$ dans 3 échantillons et de $10^{4} / \mathrm{ml}$ dans 2 échantillons.

Les cultures ont mis en évidence de 2 à 9 germes différents par échantillon, soit au total 236 germes pour les 46 échantillons.

Les principaux germes rencontrés sont les suivants :

- Groupe des staphylocoques70 fois (dont S. epidermidis, 30 fois et S.haemolyticus, 12 fois)

- Corynebactéries 36 fois

- Groupe des streptocoques 27 fois

- Enterocoques 16 fois

- Lactobacillus 8 fois

- Pseudomonas 8 fois

- Escherichia Coli 7 fois

- Ureaplasma ureolyticum 7 fois

- Gardenerella vaginalis 6 fois

- Haemophilus 4 fois

Cette étude nous confirme donc le risque de contamination du sperme lors de son expulsion à travers l'urèthre.

\section{ETUDE DE LA FLORE CUTANEO- MUQUEUSE DES ZONES GENITALES, PERIGENITALES ET DES MAINS}

FLEURETte [2] a montré la présence quasi constante de staphylocoques sur différentes territoires cutanés, en association avec d'autre germes.
En ce qui concerne les surfaces cutanées proches du pénis (pouvant donc se contaminer par le frottement des sous-vétements) et celles des bras et des mains, l'auteur rapporte les résultats qui figurent dans le tableau 1.

\section{MISE AU POINT D'UN PROTOCOLE POUR LE PRELEVEMENT}

\section{Matériel et Méthodes}

Nous avons réparti au hasard en deux groupes de 52 individus des hommes venant au laboratoire pour la première fois avec une prescription de spermoculture. Nous avons vérifié que ces patients n'avaient pas été soumis à une antibiothérapie au cours du mois précédant le prélèvement de sperme.

Les individus du groupe A ont été reçus par le personnel d'accueil du secrétariat qui leur a donné les consignes habituelles rappelées par une affiche placardée dans la cabine de prélèvement (document $n^{\circ} 1$ ) et leur a distribué une brosse à ongles à usage unique et une ampoule de gluconate de chlorhexidine.

Les individus du groupe B ont été reçus individuellement pendant quelques minutes par un médecin de l'équipe qui leur a commenté le document $\mathrm{n}^{\circ} 2$ en s'assurant de sa parfaite compréhension.

Après liquéfaction de l'éjaculat, 500 $\mu l$ de sperme ont été prélevés à l'aide d'une pipette Pasteur stérile et placés dans un tube à hémolyse stérile. Après avoir été conservés au réfrigérateur à $+4^{\circ} \mathrm{C}$, les échantillons ont été adressés au laboratoire de microbiologie et mis en culture entre 3 et 6 heures après l'éjaculation.

\section{Résultats}

Les résultats des spermocultures sont exprimés, d'une part en logarithme du nombre de germes par ml (Figure 1) et, d'autre part, en nombre de colonies détectées (Figure 2). 
Tableau 1 : Composition de la flore cutanée (d'après J.Fleurette, 1990).

\begin{tabular}{|c|c|c|c|}
\hline \multirow[t]{2}{*}{ LIEU } & \multirow{2}{*}{ 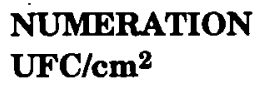 } & \multicolumn{2}{|c|}{ NATURE DES MICROORGANISMES } \\
\hline & & Dominants & Autres microorganismes \\
\hline Aine et périnée & $10^{6}$ & $\begin{array}{l}\text { S.epidermidis } \\
\text { S.hominis } \\
\text { S.haemolyticus }\end{array}$ & $\begin{array}{l}\text { Corynebacterium sp. } \\
\text { Enterobacteries } \\
\text { C.albicans }\end{array}$ \\
\hline Bras et jambes & $10^{1}-10^{2}$ & $\begin{array}{l}\text { S.epidermidis } \\
\text { S.hominis } \\
\text { S.haemolyticus }\end{array}$ & $\begin{array}{l}\text { Acinetobacter sp. } \\
\text { Micrococcus } \\
\text { Corynebacterium sp. } \\
\text { Propionibacterium sp. }\end{array}$ \\
\hline Espaces interdigitaux & $10^{3}-10^{4}$ & $\begin{array}{l}\text { S.epidermidis } \\
\text { S.hominis } \\
\text { S.haemolyticus }\end{array}$ & $\begin{array}{l}\text { Corynebacterium sp. } \\
\text { Enterobacteries } \\
\text { Levures } \\
\text { Streptococcus sp }\end{array}$ \\
\hline
\end{tabular}

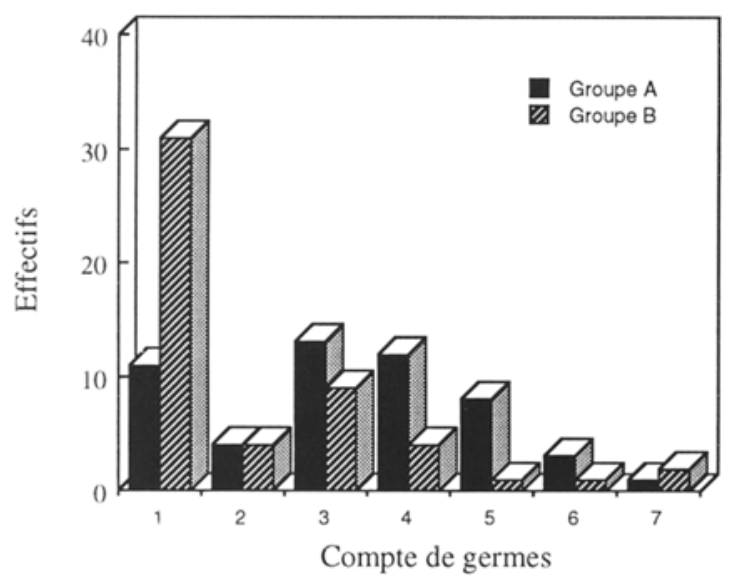

Figure 1 : Distribution en fréquence des deux groupes $A$ et $B$ pour les effectifs dans les différentes valeurs du logarithme du compte de germes.

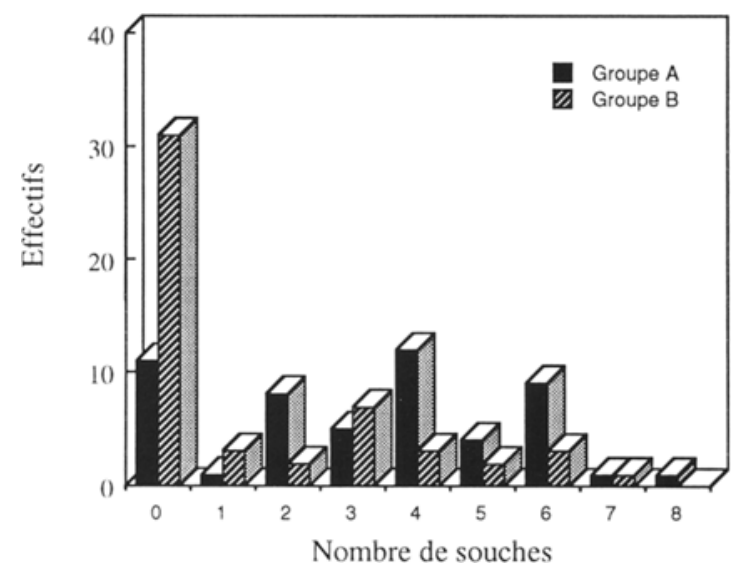

Figure 2 : Distribution en fréquence des deux groupes $A$ et $B$ pour les effectifs dans les différentes valeurs du nombre de souches.

Tableau 2 : Comparaison des deux groupes $A$ et $B$ après un regroupement des variables en trois classes : spermoculture stérile, spermoculture avec un germe dominant, spermoculture ininterprétable.

TAILLE DES EFFECTIFS

Effectifs

Groupe A (\%a)

Groupe B (\%b)

TOTAL

\begin{tabular}{lccc}
\hline Stérile & $12(23,1)$ & $31(59,6)$ & 43 \\
Un germe dominant & $14(26,9)$ & $7(13,5)$ & 21 \\
Spermoculture ininterprétable & $26(50)$ & $14(26,9)$ & 40 \\
\hline TOTAL & $52(100)$ & $52(100)$ & 104
\end{tabular}




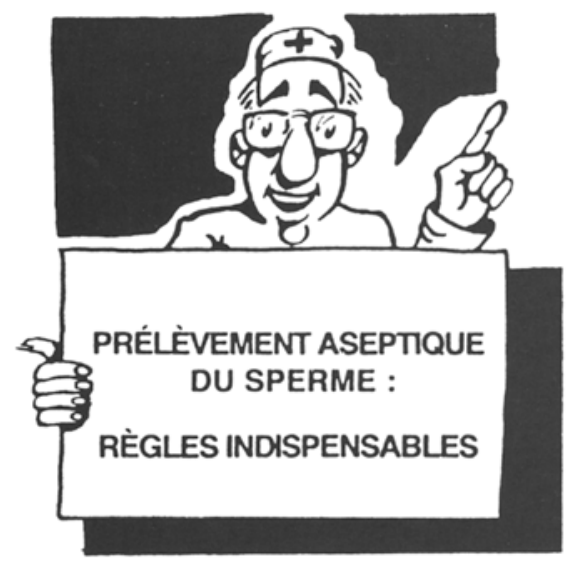

2: AU LABORATOIRE BIEN SE LAVER APRES AVOIR VIDE SA VESSIE. DEVISSER LE BOUCHON DU FLACON STERILE SANS L'OUVRIR.
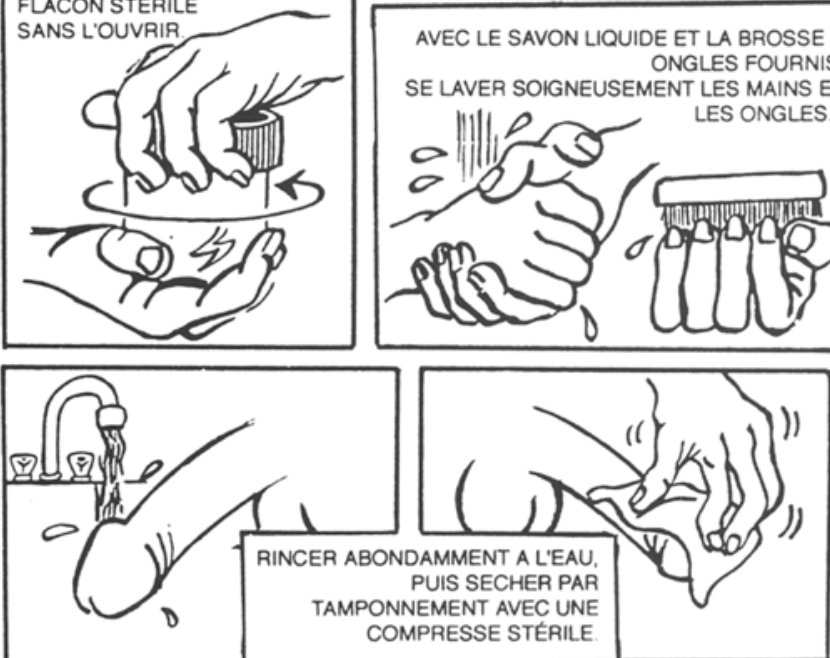

1: CHEZ VOUS DANS LA JOURNEE PRELEVEMENT.
BOIRE ET URINER BEAUCOUP

QUE FAIRE?: BUVEZ BEAUCOUP D'EAU ET URINEZ PLUSIEURS FOIS

POURQUOI? LES URINES VONT NETTOYER L'URETRE (CONDUIT COMMUN AU SPERME ET A L'URINE)

QUAND?: DES LA VEILLE DU

PRELEVEMENT PENDANT TOUTE LA JOURNEE PUIS LE MATIN BOIRE 3/4

DE LITRE DE LIQUIDE (E.AU, THE.

ETC.) URINEZ CHEZ VOUS

EN ARRIVANT AU LABORATOIRE VOUS BOIREZ A NOUVEAU 2 A 4 VERRES D'EAU ET VIDEREZ VOTRE VESSIE JUSTE AVANT UE
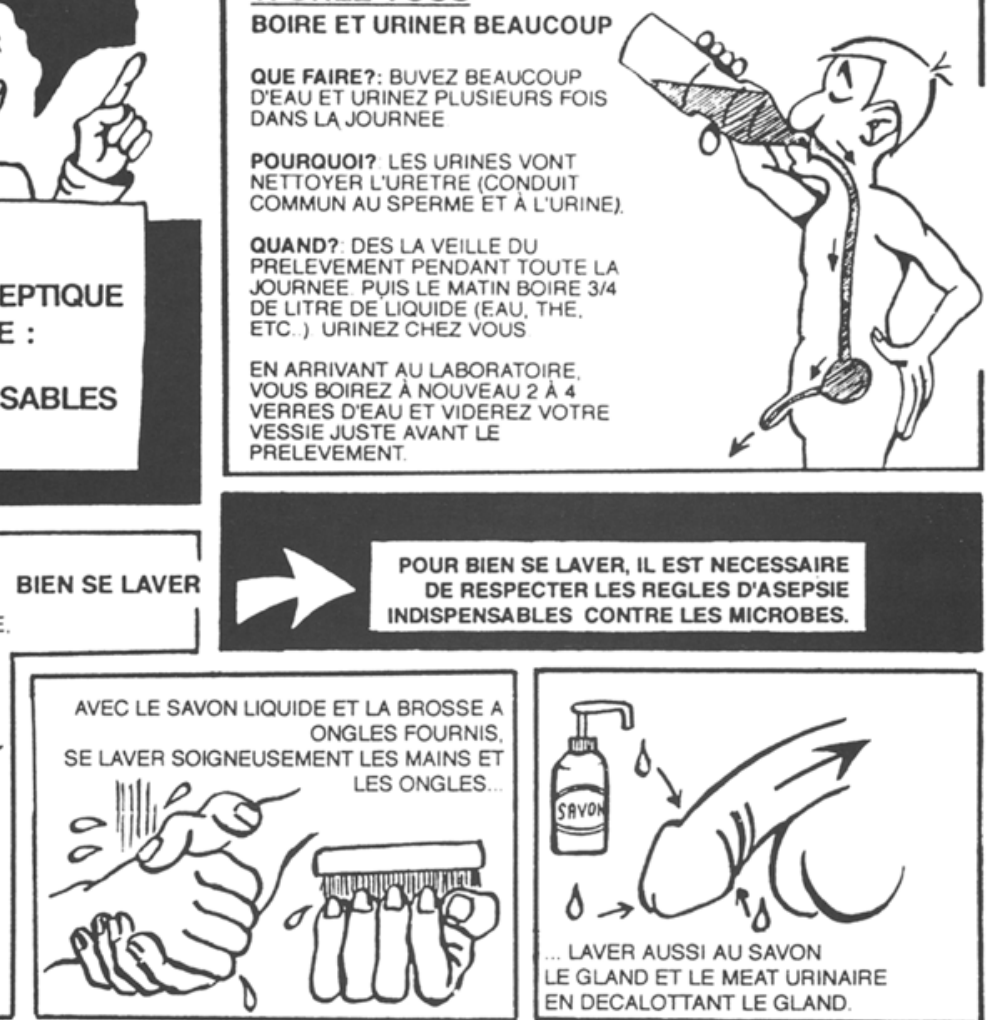

LAVER AUSSI AU SAVON LE GLAND ET LE MEAT URINAIRE EN DECALOTTANT LE GLAND

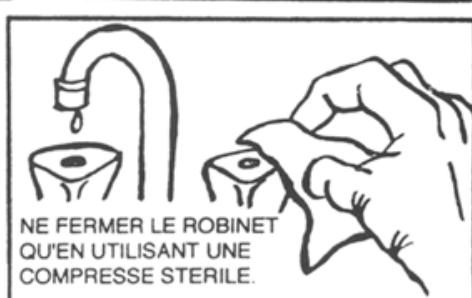

A PARTIR DE MAINTENANT, VOUS DEVEZ CONSERVER VOS MAINS PROPRES; TOUT CE QUE VOUS POURREZ TOUCHER EST SUCEPTIBLE DE CONTENIR DES GERMES CONTAMINANTS QUI PEUVENT SOUILLER VOTRE PRÉLEVEMENT.
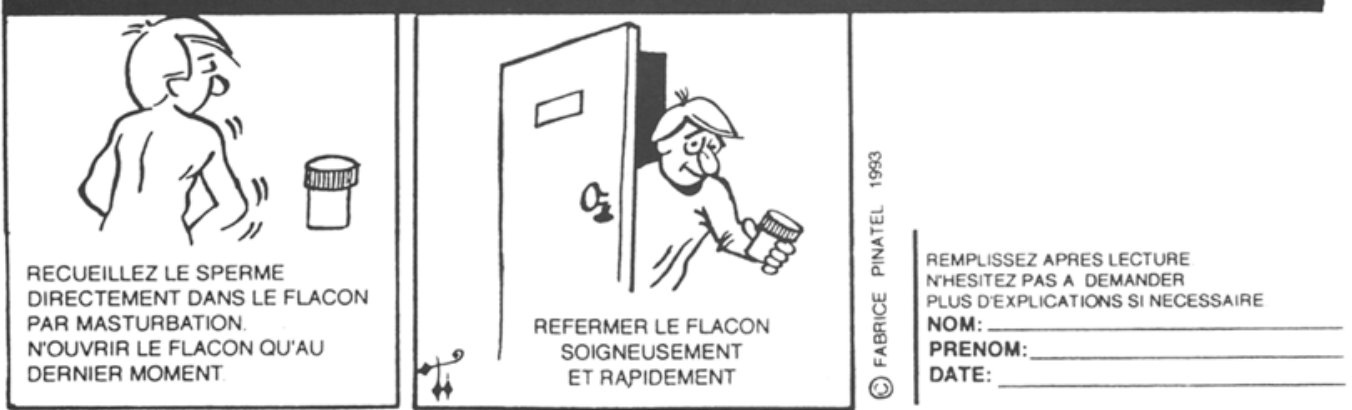

Figure 3 : Bande dessinée remise aux consultants (Ce document a été édité grâce à l'obligeance des laboratoires SERONO). 
Nous estimons qu'un germe est dominant s'il est au moins 10 fois plus représenté que les autres. Nous procédons alors à son identification.

Sur ces bases, nous avons déterminé 3 classes de spermocultures (Tableau 2):

- classe 1 :culture stérile ( $<10^{2}$ germes $/ \mathrm{ml}$ )

- classe 2 :présence d'un germe dominant

- classe 3 : présence de plusieurs souches bactériennes sans dominance. Le sperme est alors considéré comme contaminé et les résultats sont ininterprétables.

Nous pouvons remarquer que le groupe B comporte plus du double de spermocultures stériles $(59,6 \%)$ que le groupe A $(23,1 \%)$. Le nombre de spermocultures ininterprétables est réduit de moitié dans le groupe $B$ $(26,9 \%)$ par rapport au groupe A $(50 \%)$.

\section{Discussion}

Même en l'absence d'infection urinaire, l'urèthre contient des germes et les zones cutanées périgénitales et les mains en comportent également. Tous ces germes

\section{Document $n^{\circ} 1$ : Consignes habituellement prescrites pour les prélèvements de sperme et adressées au groupe A.}

PRECAUTIONS DE PRELEVEMENT A OBSERVER AVANT SPERMOCULTURES, IAC, FECONDATION IN VITRO, DON DE SPERME :

\section{Uriner}

2. Dévisser le flacon à prélèvement sans ôter le couvercle

3. Faire un soigneux lavage des mains et des ongles avec la brosse au savon liquide (produit $n^{\circ}$ 1) *

4. Faire une toilette intime minutieuse en décalottant le gland (compresse stérile + produit $\left.\mathrm{n}^{\circ} 2\right)^{* *}$

5. Rincer soigneusement

6. Pratiquer le prélèvement par masturbation

* Chlorhexidine 4\% (HIBISCRUB)

** Gluconate de chlorhexidine (GIFRER) peuvent être retrouvés dans le sperme lors d'un recueil effectué sans précaution spéciale.

Nous avons pu montrer qu'une information personnalisée, en entretien particulier, durant quelques minutes, permet de motiver le patient pour réaliser un prélèvement dans de bonnes conditions d'asepsie. Le nombre d'examens ininterprétables, et devant par conséquent être renouvelés, est réduit de moitié ; se trouve également réduit le nombre d'antibiothérapies abusives.

Cependant, dans la pratique quotidienne, l'équipe médicale ne dispose pas toujours de temps et de locaux suffisants pour un entretien avec les patients qui viennent pour une spermoculture. Nous avons alors décidé de leur faire remettre par le médecin prescripteur un document explicatif en bande dessinée (Figure 3). Ce document que nous avons nous-même élaboré, semble bien apprécié par les consultants.

\section{CONCLUSIONS}

Après avoir vérifié la présence quasi constante de germes dans la première miction matinale, nous avons conclu à l'intérêt de faire boire abondamment les consultants adressés au laboratoire pour une spermoculture, afin de provoquer plusieurs mictions avant le prélèvement de sperme.

Les observations publiées par d'autres auteurs nous ont montré la fréquence et l'importance de la présence des nombreux germes à la surface des zones périgénitales et des mains, ce qui nous a conduits à demander aux patients une toilette très minutieuse de ces régions cutanées. Nous proposons une information facile des consultants à l'aide d'une simple bande dessinée.

L'étude des résultats ainsi obtenus nous montre que l'on peut réduire considérablement le nombre des examens ininterprétables. 
Document $n^{\circ} 2$ : Protocole de prélèvement pour les spermocultures du groupe $B$.

\section{PROTOCOLE DE PRELEVEMENT POUR SPERMOCULTURES}

Les mains, la peau, les muqueuses et les objets sont normalement porteurs de microbes. Afin d'éviter de souiller votre prélèvement avec ces germes, il vous faut respecter quelques règles d'asepsie indispensables.

En arrivant au laboratoire, vous devez boire au moins 2 à 4 grands verres d'eau. Puis vous viderez votre vessie une première fois avant l'enregistrement de votre dossier et une deuxième fois juste avant votre prélèvement.

Vous allez pratiquer votre prélèvement de la façon suivante :

1. Dévissez avec précautions le bouchon du flacon stérile et laissez le posé sur ce récipient jusqu'au dernier moment. Lavage soigneux au savon antiseptique des mains, du gland et du méat urinaire ; rinçage abondant puis séchage par tamponnement avec une compresse stérile.

A partir de maintenant, vous devez conserver vos mains propres, tout ce que vous pourrez toucher est susceptible de contenir des germes contaminants qui peuvent souiller votre prélèvement.

2. Recueillez le sperme directement dans le flacon par masturbation. Il vous est fourni une compresse imprégnée d'huile de vaseline stérile, utilisez la si une lubrification vous est nécessaire. Refermer le flacon soigneusement et rapidement.Si vous respectez ces consignes, vous aurez un prélèvement de bonne qualité, indispensable pour la bonne interprétation des résultats.

Il est possible que ce texte ne vous semble pas très clair. Lisez le plusieurs fois et n'hésitez pas à demander des explications si vous le jugez nécesaire.

\section{REFERENCES}

1. BOUCHER P. : Etude de la contamination du plasma séminal par la flore physiologique. Interprétation des spermocultures. Thèse de Doctorat d'Etat en Pharmacie. Université Lyon I, 1993.

2. FLEURETTE J. : Taxonomie et écologie des staphylocoques coagulases négatifs. Médecine et Maladies Infect., 1990, hors série, $20: 6-15$.

\section{ABSTRACT}

A protocol of semen collection for bacteriological screening.

M.C. Pinatel, P. Boucher, Y. Gille, F. Pinatel

*Laboratoire de Biologie de la Reproduction. Hôpital E. Herriot Place d'Arsonval 69003 Lyon ; **Laboratoire de Microbiologie de l'Hôpital de l'Antiquaille Lyon; ***Faculté des Arts Plastiques de Saint-Etienne

The purpose of the protocol is to put the patients into contribution on an aseptic way of obtaining semen in order to avoid renewal of numerous uninterpretable tests due to the presence of contaminating germs.

This protocol of semen collection in aseptic conditions is recommended before IVF and artifical insemination.

Key Words : Semen, bacteriological culture of semen, semen collection. 